\title{
Re: Peri-operative Outcomes and Predictors of Mortality in COVID-19-Positive Patients with Hip Fractures: A Multicentre Study in the UK
}

\author{
Parth Bansal $^{1}{ }^{1}$. Devendra Kumar Chouhan ${ }^{1} \cdot$ Sandeep Patel ${ }^{1} \cdot$ Mandeep S. Dhillon $^{1}$
}

Received: 9 March 2021 / Accepted: 7 May 2021 / Published online: 17 May 2021

(c) Indian Orthopaedics Association 2021

To the Editor,

The article by De et al. [1] on peri-operative outcomes and mortality in COVID-19-positive patients with hip fractures holds great relevance with the current worldwide situation with respect to the pandemic. Hip fractures especially those occurring in the elderly (fragility fractures) are a leading cause of morbidity and mortality in orthopaedic patients. We would like to give our opinion about the following points:

1. In the study protocol, COVID-19 tests were done only for patients who fulfilled clinical or radiological criteria of COVID-19 infection. The inclusion of only symptomatic patients for testing would lead to underreporting of infection in this cohort of patients. Patients who were asymptomatic would also logically have lower rates of pulmonary complications and mortality, and their exclusion would lead to higher mortality being attributed to COVID-19. A possible solution would be to label the study sample as 'symptomatic COVID-19 positive patients'.

2. The study protocols mentioned that patients were operated in 'COVID-designated theatres'. But results from

This comment refers to the article available online at https://doi. org/10.1007/s43465-020-00272-7.

Parth Bansal

parthbansa193@gmail.com

Devendra Kumar Chouhan

drdevnim@gmail.com

Sandeep Patel

sandeepdrpatelortho@gmail.com

Mandeep S. Dhillon

drdhillon@gmail.com

1 Department of Orthopaedics, PGIMER Chandigarh, Chandigarh 160012, India
Table 1 have divided the patients into two groups-those who were operated in 'COVID-designated theatres' and those who were operated in 'clean trauma theatre'. We request the authors to provide a clarification for this point, with respect to how allocation was done for patients to any specific theatre. We also request the authors to give their opinion on where they feel these patients should be operated, both from the patients' point of view and in view of restricting transmission to healthcare workers.

3. An international multicentre cohort study conducted in over 35 hospitals has found that 30-day mortality in patients undergoing surgery with COVID-19 infection was $23.8 \%$ (268 of 1128) [2]. They opined that higher thresholds should be set for deciding on operative intervention, especially in the older age group. They also felt that non-operative treatment measures should be used to delay or avoid surgery in high-risk patients. The treatment protocol in our institution for hip fractures has been to treat them in the same category as other closed fractures-to be operated once the patient is COVID negative. We feel that further research is needed, to ascertain whether non-operative treatment has better/ comparable outcomes in COVID positive patients specifically, or whether we need to develop more protocols and resources for urgent treatment of these patients.

We would like to thank the authors for their pioneering work in the field of hip fractures in COVID-19-positive patients, and await their comments.

Funding No funding was required. 


\section{Declarations}

Conflict of interest The authors declare no conflict of interest.

Ethical approval This article does not contain any studies with human or animal subjects performed by the any of the authors.

Informed consent For this type of study informed consent is not required.

\section{References}

1. De, C., Wignall, A., Giannoudis, V., Jimenez, A., Sturdee, S., Aderinto, J., Pandit, H., Palan, J., \& Gulati, A. (2020). Peri-operative outcomes and predictors of mortality in COVID-19 positive patients with hip fractures: a multicentre study in the UK. The Indian Journal of Orthopaedics, 54, 1-11.

2. COVIDSurg Collaborative. (2020). Mortality and pulmonary complications in patients undergoing surgery with perioperative SARS-CoV-2 infection: an international cohort study. Lancet, 396, $27-38$.

Publisher's Note Springer Nature remains neutral with regard to jurisdictional claims in published maps and institutional affiliations. 